A. Pramesh Rao, G. Swarup and Gopal-Krishna, eds.

\title{
The SEDs Database to Study Evolution of Radio Galaxies
}

\author{
O.V.Verkhodanov, A.I.Kopylov, O.P.Zhelenkova, N.V.Verkhodanova, \\ V.N.Chernenkov, Yu.N.Parijskij, N.S.Soboleva and A.V.Temirova \\ Special Astrophysical Observatory, Nizhnij Arkhyz, Russia, 369167
}

\begin{abstract}
The project of the informational system creation on the problem of evolution of radio galaxies is described. This system, being developed at present, will allow a user to operate with simulated curves of spectral energy distributions (SED) and to estimate ages and redshifts by photometrical data. Planned modes of access, formats of output result and additional functions are described.
\end{abstract}

The last few years have changed our view on the evolution of galaxies: the most distant galaxies (i.e. systems having stellar population) have been found at $z=6.68$ (Chen et al., 1999) and radio galaxies at $z=5.2$ (van Breugel et al., 1999). To understand deeper a situation with a stellar population of host galaxies and to check various mordern models it is very important to have a capability to detect correctly an age of galaxies. The labour intensity of obtaining statistically significant high-quality data on distant and faint galaxies and radio galaxies forces one to look for simple indirect procedures in the determination of redshifts and other characteristics of these objects. With regard to radio galaxies, even photometric estimates turned out to be helpful and have so far been used (McCarthy, 1993). Over the last few years the three models: PEGASE (Project de'Etude des Galaxies par Synthese Evolutive (Fioc and Rocca-Volmerange, 1997)), Poggianti (1997) and GISSEL'96 (Bruzual, Charlot, 1996), have been extensively used, in which an attempt has been made to eliminate the shortcomings of the previous versions.

In the "Big Trio" experiment (Parijskij et al., 1996) we also attempted to apply these techniques to distant objects of the RC catalogue with ultra steep spectra (USS). Color data for nearly the whole basic sample of USS FR II (Fanaroff and Riley, 1974) RC objects have been obtained with the $6 \mathrm{~m}$ telescope of SAO RAS. To accelerate a procedure of age (and photometric redshift) estimation we have begun a project "Evolution of radio galaxies", supported by the Russian Foundation of Basic Research (grant No. 99-07-90334), which has to allow a user to obtain age and photometric redshift estimations.

\section{Description of a system}

This system, being developed at present, will allow a user to operate with simulated curves of spectral energy distributions (SED) to estimate ages and redshifts by photometral data. Authors use SEDs of three models: (1) PEGASE, (2) Poggianti(1996)) and (3) GISSEL'96. for different types of galaxies. 
The system will be situated on the special Web-server sed.sao.ru unifying various type resources, including specialized Internet protocol daemons (for the FTP, HTTP, e-mail support) and the designed software permitting a user to operate with the SED curves. Requesting and filling in the standard HTMLforms a user will be able to select different types of curves or trust to do this to a computer by the $\chi^{2}$ method. The input forms contain information about input filters or wavelengths and corresponding magnitudes.

There will be a possibility to detect the age of galaxies in two ways: (1) by fixed (known) redshift; (2) and when variable (unknown) redshift is. In latter the redshift will be estimated too. The estimation of ages and redshifts is performed by way of selection of the optimum location on the SED curves of the measured photometric points obtained when observing radio galaxies in different filters. We use the already computed table SED curves for different ages. From the whole collection of curves, we select the ones on which the sum of the squares of the discrepancies turn out to be minimal for the given observations of radio galaxies.

There will be a chance to use infrared maps for absorption estimates in a case when magnitudes are not corrected for absorption. In order to take account of the absorption, we apply the maps (as FITS-files) from the paper "Maps of Dust IR Emission for Use in Estimation of Reddening and CMBR Foregrounds" (Schlegel et al., 1998).

Another possibilities are supposed to be supported in our system: (a) sorted bibliographical collection of papers for different stages of radio galaxy evolution, (b) archive of radio galaxies data in various wavelength ranges (both observed in Special astrophysical observatory and taken from Internet) and (c) search for information about radio galaxies using the largest data bases NED, CATS, LEDA et al.

The e-mail access to the basic procedures is organized in this system too. Special e-mail forms are prepared to reflect input flags of low level procedures. FTP access is organized to give a user a possibility to obtain SEDs models recorded in separate files. A result is supposed to be written in ASCII tables and PS-figures and can be sent to users.

\section{References}

van Breugel W. et al. ApJ, 518, L61, astro-ph/9904272.

Chambers K. \& Charlot S., Astroph.J. Let.,1990. 348, L1,

Chen H.-W., Lanzetta, K. M., \& Pascarelle, S. 1999, Nature, 398, 586.

Fanaroff B.L. \& Riley J.M. 1974, MNRAS, 167, 31.

Fioc M. \& Rocca-Volmerange B. 1997, A\&A, 326, 950.

McCarthy P.J. 1993. ARA\&A, 31, 639.

Parijskij Yu.N., et al. 1996, Bull.SAO, 40,5,

Poggianti B.M. 1997, A\&A, 122, 399.

Schlegel D., Finkbeiner D., \& Davis M. 1998, ApJ, 500, 525.

Verkhodanov O.V. et al. 1999., Bull.SAO, 48, 41. 